\title{
Considerações sobre a seleção de Áreas Protegidas, o Planejamento Sistemático da Conservação e a escala de abordagem
}

\author{
Larissa Monteiro Rafael ${ }^{(a)}$ \\ ${ }^{(a)}$ Departamento deGeografia (Itabaiana), Universidade Federal de Sergipe,llarissarafaell@ gmail.com
}

\section{EIXO:BIOGEOGRAFIA, MANEJO DE ÁREAS NATURAIS E ROTEGIDAS: CONSERVAÇÃO DA BIODIVERSIDADE}

\begin{abstract}
Resumo:
Até a década de 1970 as metodologias voltadas à seleção de Áreas Protegidas eram pouco sistematizadas, acarretando na criação de áreas isoladas e sem integração regional. A partir de perspectivas mais recentes, essas áreas deixam de exercer uma função isolada para comporem pilares sobre os quais a integração regional para a conservação da biodiversdiade seja realizada. Nesse âmbito, o Planejamento Sistemático da Conservação visa apresentar um estrutura para seleção e desenho das APs a partir de regiões de planejamento. Discute-se aqui o papel da escala nessa abordagem regional de integração, considerando sua natureza de síntese e a necessidade de ações de conservação em uma escala de detalhe.
\end{abstract}

Palavras chave: Planejamento da Conservação; Escalas da Paisagem; Conservação da Biodiversidade.

\section{Introdução}

Um dos instrumentos importantes para a conservação da biodiversidade diz respeito à criação de Áreas Protegidas (AP). Originalmente configurada para garantir a proteção dos ecossistemas idílicos que serviriam de reduto de contato com a natureza para as populações urbanas, sua concepção vem sofrendo reconfigurações incluindo desde a necessidade de conservação da diversidade biológica até, mais recentemente, a justiça socioambiental para as populações impactadas por essa criação.

A criação de áreas protegidas ainda representa um significativo desafio ao planejamento sistemático da conservação. Ainda que o incremento dessas áreas não represente em si a solução para a conservação da biodiversidade, ela assume a função principal, responsável por ser representativa dessas áreas (MARGULES e PRESSEY, 2000; PRESSEY et al., 1993).

No Brasil, não existe uma definição sobre AP absorvida pelo aparelho legislativo. O instrumento mais eficaz para a conservação dos remanescentes florestais diz respeito ao Sistema Nacional de Unidades de Conservação da Natureza (SNUC), sendo responsável por criar, implementar e gerir as Unidades de Conservação (UCs) de forma a garantir seu planejamento e administração de forma integrada (BRASIL, 2000). Assim, os instrumentos regulatórios e os 
planos de ação são deficientes no estabelecimento de uma articulação para o planejamento da conservação.

Paralelamente, essas estratégias regionais para conservação estabelecem articulações deficientes entre os componentes interatuantes em escala regional, geralmente materializados na forma de planos, e em escala local, a partir das ações de conservação. Discute-se aqui o papel da escala nessa abordagem regional de integração, considerando sua natureza de síntese e a necessidade de ações de conservação em uma escala de detalhe.

\section{2. Áreas Protegidas e iniciativas de integração regional}

Os registros históricos brasileiros indicam que no período colonial, de reinado e imperial os dirigentes tomaram algumas iniciativas destinadas à proteção, à gestão ou ao controle de determinados recursos naturais (BENSUSAN, 2006; MEDEIROS, 2006). Em 1808, por exemplo, Dom João IV criou o Jardim Botânico do Rio de Janeiro para servir como uma área de conservação ex situ responsável por aclimatar as especiarias do Oriente. Ele representava uma forma de competição com o mercado chinês de plantação de chá preto (Camelliasinensis) (CROSBY, 2011). Essas iniciativas pristinas, entretanto, centravam-se em determinados recursos não acarretando necessariamente em uma delimitação territorial. A noção só seria modificada quando os efeitos da degradação biológica e física da paisagem foram percebidos em uma esfera global e, mais tardiamente, nacional (MEDEIROS, 2006).

No país não existe uma definição legal para área protegida, mas, de acordo com o glossário disponível na página eletrônica do Ministério do Meio Ambiente (MMA), áreas protegidas correspondem às "áreas de terra e/ou mar especialmente dedicadas à proteção e manutenção da diversidade biológica, e de seus recursos naturais e culturais associados, manejadas por meio de instrumentos legais ou outros meios efetivos" (MMA, s/d). Observa-se que a definição brasileira muito se assemelha àquela apresentada pela UICN - instituição que tem suas categorias de área de proteção adotadas como padrão global para definição e reconhecimento de áreas protegidas (DUDLEY, 2008). Para essa definição, apenas as Unidades de Conservação se enquadram, uma vez que são as únicas com fins especialmente voltados à conservação da natureza. Considerado, porém, área de proteção em um sentido amplo de contribuição à conservação, o número de categorias de APs se expande, podendo ser inclusas também as Reservas Legais, as Áreas de Preservação Permanente, as Terras Indígenas e os sítios de proteção criados a partir de convenções e tratados internacionais - Reservas da Biosfera, Sítios do Patrimônio da Humanidade e Sítios Ramsar(MEDEIROS, 2006; MEDEIROS e GARAY, 2006). 
Do ponto de vista legal, a criação do Código Florestal (BRASIL, 1934) significou a possibilidade do estabelecimento de áreas protegidas para o país (BENSUSAN, 2006; MEDEIROS, 2006; RYLANDS e BRANDON, 2005). Seguindo a tendência internacional, as primeiras áreas protegidas legalmente institucionalizadas corresponderam aos parques nacionais. Os interesses de conservação, contudo, eram diferentes daqueles americanos que contrapunham civilização à natureza. Na experiência brasileira, buscou-se melhorar a eficiência na exploração da natureza para a promoção do "progresso" (PÁDUA, 2004). Assim, a história de criação dessas áreas evidencia um objetivo primeiramente ligado a questões territoriais e, secundariamente, à preservação da biodiversidade. De acordo com Castro-Junior e colaboradores (2009) a prioridade na escolha das áreas estava relacionada ao grau de concentração populacional e de atividades humanas e, nesse sentido, muitas unidades incrementadas são, ainda hoje, espaços de conflitos territoriais em diferentes escalas. Existe o interesse do Estado no controle do território e o desinteresse de setores ligados ao capital produtivo e de comunidades alocadas na região as quais possuem pouca possibilidade de participação no processo de demarcação das unidades.

Observa-se que, no contexto da conservação da natureza, o Brasil ainda carece de um sistema integrado que sirva para essa finalidade. Atualmente o instrumento mais eficaz nesse sentido corresponde às diretrizes e ações do Sistema Nacional de Unidades de Conservação (SNUC), quando se verifica o descaso com outros instrumentos que também deveriam ser empregados para essa finalidade, uma vez que o Brasil compreende em seu território uma importante diversidade biológica e cultural que devem ter sua existência garantida.

Assim, para a realidade brasileira, áreas de proteção como um instrumento ativo de conservação da natureza, regido por um arcabouço diretivo e fiscalizador, acaba se restringindo ao incremento de UCs. Do ponto de vista prático, as demais iniciativas acabam se tornando ações isoladas sem conexões com um planejamento sistemático. As UCs cridas não serviam à proteção para futuros impactos como se via no modelo americano, mas à limitação de impactos imediatos. Assim, muitas dessas áreas foram estabelecidas "em meio a importantes conflitos territoriais e de acesso a recursos" (GUERRA e COELHO, 2009, p. 39).

No contexto dos países em desenvolvimento, o incremento das APs reflete a realidade associada às contradições e desigualdades sociais (DIEGUES, 2008; GOMEZ-POMPA e KAUS, 1992). Ou seja, não se trata apenas de estabelecer metas para conservação da biodiversidade uma vez que sua manutenção está associada à deterioração social. Pensar em um planejamento sistemático da conservação para os ecossistemas brasileiros exige maior integração com os diversos interesses que emergem a partir da delimitação do uso da terra 
Pensando nessa integração das áreas protegidas (stricto senso) com as diversas escalas de planejamento e gestão do território, novas iniciativas vêm sendo motivadas. Do ponto de vista da gestão territorial para conservação, quatro ferramentas fortalecedoras dessa integração vêm se destacando na agenda governamental:

a) o Plano Estratégico Nacional de Áreas Protegidas (PNAP) - instituído através do Decreto 5.758, de abril de 2006 é uma resposta ao Programa de Trabalho para Áreas Protegidas da CDB (Decisão VII/28) adotado por países signatários durante a Sétima Conferência das Partes - COP 7 da Convenção sobre Diversidade Biológica (CDB). Seu objetivo é "orientar as ações que se desenvolverão para o estabelecimento de um sistema abrangente de áreas protegidas ecologicamente representativo, efetivamente manejado, integrado a áreas terrestres e marinhas mais amplas, até 2015"(BRASIL, 2006). Observa-se que esse "sistema abrangente de áreas protegidas" enfoca prioritariamente o SNUC, as terras indígenas e os territórios quilombolas não inserindo as reservas legais e áreas de proteção permanente previstas pelo Código Florestal em sua política. De acordo com Castro-Junior et al.(2009), o plano deve se tornar um importante instrumento para a gestão de UC;

b) os mosaicos de UC - constituem um conjunto de UCs de categorias diferentes ou não, próximas, justapostas ou sobrepostas, e outras APs públicas ou privadas (BRASIL, 2000). De acordo com Jorge-Pádua (2011), esse instrumento previsto no SNUC vem estimulando a gestão integrada e participativa das UCs, integrando os diversos setores da sociedade e, ainda, otimizando a gestão dos recursos financeiros e humanos.

c) a Reserva da Biosfera - também prevista na Lei que institui o SNUC, objetiva: "preservação da diversidade biológica, o desenvolvimento de atividades de pesquisa, o monitoramento ambiental, a educação ambiental, o desenvolvimento sustentável e a melhoria da qualidade de vida das populações" (BRASIL, 2000, Art. 41). Constituída por áreas-núcleo, zonas de amortecimento e zonas de transição, acrescentam mais categorias de manejo à soma prevista para as UCs. Porém sua aplicação não tem incentivado modificações nas práticas socioespaciais, uma vez que as restrições de determinadas práticas previstas nesse ordenamento territorial não ocorrem (JORGE-PÁDUA, 2011);

d) os Corredores ecológicos - adoção de técnicas da biologia da conservação e estratégias de planejamento e gestão socioambiental de forma compartilhada e participativa, representando não unidades políticas ou administrativas, mas áreas geográficas para fins de planejamento e conservação (AYRES et al., 2005). Abrangem conexões entre UCs federais, estaduais e municipais e terras indígenas visando fortalecer as áreas de proteção já 
existentes (BENSUSAN, 2006; RYLANDS e BRANDON, 2005). Essa estratégia demonstra ampliar a efetividade na implementação do SNUC integrando essas áreas a uma estratégia de gestão de paisagens (LIMA et al., 2011).

O próprio SNUC reconhece e consagra em seu texto a importância dessas ferramentas para o processo de gestão das áreas protegidas (à exceção do Plano Estratégico Nacional de Áreas Protegidas). Na prática, porém, as experiências e os resultados são ainda muito pouco numerosos e bem avaliados (MEDEIROS, 2006).

Do ponto de vista empírico, o instrumento brasileiro mais eficaz na conservação dos ecossistemas diz respeito ao Sistema Nacional de Unidades de Conservação (BENSUSAN, 2006; MEDEIROS, 2006; MORSELLO, 2006), que define e regulamenta as categorias de UC nas instâncias federal, estadual e municipal (BRASIL, 2000). Esse sistema as delimita a partir de dois grupos: (1) proteção integral, com a conservação da biodiversidade como objetivo principal; (2) áreas de uso sustentável, com objetivo na regulação das várias formas dos recursos naturais e, neste caso, a conservação da diversidade biológica em segundo plano.

Um dos principais instrumentos que orientam as ações nas Unidades de Conservação é o plano de manejo. A realidade das Áreas Protegidas brasileiras legalmente criadas, contudo, conta com a ausência/ineficiência do suporte de um plano de manejo adequado ao contexto local e articulado com a escala regional (MEDEIROS e YOUNG, 2011).

\section{Planejamento Sistemático da Conservação da Biodiversidade}

Para que uma AP seja delimitada, dois são os critérios observados: o design e a localização. Esse primeiro vem sendo amplamente discutido, já a localização "ou seleção de reserva, está atualmente recebendo atenção crescente" (PRESSEY et al., 1993, p. 124 tradução nossa). Até a década de 70 princípios científicos voltados à seleção de áreas protegidas eram pouco organizados, acarretando em áreas criadas sem integração, de modo isolado (SULLIVAN e SHAFFER, 1975). Um exemplo diz respeito à Teoria de Biogeografia de Ilhas - desenvolvida por McArthur e Wilson (1967) para explicar a ocorrência de espécies em ilhas oceânicas em relação à taxa de colonização e extinção ao longo do tempo - adotada para fragmentos continentais como aplicação potencial para a conservação biológica. Propôs-se a aplicação dos princípios das ilhas oceânicas para as "ilhas" de fragmentos, sugerindo que o desenho das APs poderia apresentar uma redução da taxa de extinção (DIAMOND, 1975). Ainda contraditória quanto a possibilidade de aplicação aos fragmentos continentais, alguns autores acreditam que,

\footnotetext{
1 “or reserve selection, is currently receiving increased attention" (PRESSEY et al., 1993, p. 124)
} 
por dar ênfase a diversidade de espécies, acaba se limitando ao desenho de reservas, se distanciando de um planejamento sistemático.

De acordo com Pressey(1994), os sistemas de áreas protegidas dificilmente são desenhados com o objetivo de conservação sistemática da diversidade biológica, não incluindo todas as espécies que o local necessita. Desse modo, ainda que as áreas de proteção representem a prática mais recorrente no sentido de conservação da biodiversidade, estudos vêm indicando que elas apresentam maiores possibilidades de exercerem seu papel quando inclusas em estratégias espaciais integradoras inseridas no contexto do planejamento da conservação (EKEN et al., 2004; MARGULES e PRESSEY, 2000; OLSON e DINERSTEIN, 1998) e considerando aspectos ecológicos, econômicos e político-institucionais (MORSELLO, 2006). São exemplos dos aspectos ecológicos iniciativas como os hotspots de biodiversidade (Cf.: MYERS, 2003; MYERS et al., 2000), a delimitação de ecorregiões(Cf.: BAILEY, 2014; OLSON et al., 2001; OLSON e DINERSTEIN, 1998) e as áreas-chave da biodiversidade (Cf.: EKEN et al., 2004; LANGHAMMER et al., 2007).

No âmbito regional uma estratégia espacial que vem se destacando e integrando as diversas abordagens ecológicas e sociais, delimitando um arcabouço sistemático de seleção e desenho das APs, diz respeito ao Planejamento Sistemático da Conservação. O processo é delimitado em seis etapas: (1) compilação de dados da biodiversidade da região alvo do planejamento; (2) identificação dos objetivos conservacionistas; (3) revisão das áreas de conservação existentes; (4) seleção de áreas adicionais para conservação; (5) implementação de ações conservacionistas; (6) manutenção dos valores necessários à conservação (MARGULES e PRESSEY, 2000). Essa e outras iniciativas - como a da análise global de lacunas da rede de áreas protegidas (Cf.: RODRIGUES et al., 2003) - representam um avanço significativo na seleção de APs e vem favorecendo ao aumento crescente de abordagens para o planejamento sistemático da conservação da biodiversidade (BENSUSAN, 2006).

\section{Escala de Síntese e de Detalhe nas regiões de planejamento}

Uma limitação da abordagem sistemática diz respeito à ausência da identificação de alvos para ações de conservação em uma escala de detalhe prevalecendo a escala de síntese (EKEN et al., 2004). Ou seja, existe uma abordagem definida para a escala regional/global, mas sua articulação com a escala local permanece deficiente. Esse quesito é evidenciado pelas lacunas de informações sociais e biológicas básicas das áreas de proteção complicando a seleção do mais adequado tamanho, formato e categoria a ser atribuída à reserva (BERNARD, BARBOSA e CARVALHO, 2011; SODHI et al., 2010). 
A dificuldade em articular a escala regional com a local implica na consulta às comunidades locais que habitam as APs mapeadas ou o seu entorno, apontadas como atores essenciais na conservação ou degradação dessas áreas. Considera-se que o estabelecimento dessas áreas acarreta em problemas de caráter político, econômico e social, não se reduzindo somente à proteção da biodiversidade (CHIMÈRE-DIAW, 2008; DIEGUES, 2008; SODHI et al., 2010). De acordo com Diegues (2008), esses entraves podem ser reunidos em três conjuntos: (1) Tipologia e características das AP que nem sempre são adequadas ao contexto social da região; (2) Impacto político-territorial e fundiário tendo em vista a extensão e quantidade das APs em regiões densamente povoadas e em governos que não avaliam adequadamente os custos da manutenção desse sistema; (3) Impacto social, étnico e ético associado à expulsão dos moradores, acarretando em conflitos no âmbito das AP e sua ineficiência quanto à conservação biológica. Assim, concomitantemente às novas perspectivas de integração espacial dos esforços conservacionistas também questões políticas, econômicas, sociais e étnicas vem compondo esse arcabouço (DAVENPORT e RAO, 2002; TERBORGH e SCHAIK, 2002).

Frequentes esforços para assegurar que as comunidades locais se beneficiem das áreas protegidas envolvem abordagens como conservação e projetos de desenvolvimento integrados, abordagens de gestão inclusiva e a criação de oportunidades para a conservação da biodiversidade na mais ampla paisagem rural, na forma de áreas de conservação comunitária (SCHERL et al., 2006). No âmbito local as metodologias participativas vêm se destacando no que diz respeito à coleta de dados tanto do meio físico quanto social. São exemplos dessa abordagem a Etnobiologia(Cf.: ALBUQUERQUE et al., 2013; POSSEY e OVERAL, 1990), o SIG Participativo (Cf.: BERNARD, BARBOSA e CARVALHO, 2011; BOURGOIN, 2012; RAMBALDI et al., 2007; SIEBER, 2006; WEINER, HARRIS e CRAIG, 2002) e a investigação de processos de formação de condutas e de orientação das comunicações sociais (BUIJS et al., 2011; FORRESTER et al., 2015; GALLINI, 2005; REIGOTA, 2010).

Observa-se uma crescente tendência em se abordar problemas ambientais e de desenvolvimento de forma sistêmica (FORRESTER et al., 2015) tanto no que diz respeito ao Planejamento Sistemático da Conservação quanto à inclusão de temas sociais para contribuir à conservação da biodiversidade. Reconhece-se, assim, a necessidade de metodologias envolvendo mais de uma disciplina (FORRESTER et al., 2015) e as limitações tanto do ponto de vista metodológico referente a cada um desses ramos científicos quando a conjuntura social presente nessas contradições. 


\section{Conclusão}

Observa-se que, no contexto da conservação da natureza, o Brasil ainda carece de um sistema integrado que sirva para essa finalidade. Para a realidade brasileira, áreas de proteção como um instrumento ativo de conservação da natureza, regido por um arcabouço diretivo e fiscalizador, acaba se restringindo ao incremento de Unidades de Conservação. Do ponto de vista prático, as demais iniciativas acabam se tornando ações isoladas sem conexões com um planejamento sistemático.

Compreende-se, ainda, que essas estratégias de planejamento regional, uma vez que envolvem a escala de síntese, falham na articulação entre o plano e a ação. Torna-se premente a aplicação de estudos da conservação da biodiversidade que busquem a integração regional das áreas protegidas, mas que considerem uma abordagem holística dos fenômenos.

\section{Bibliografia}

ALBUQUERQUE, U. P.; SILVA, J. S.; CAMPOS, J. L. A.; SOUSA, R. S.; SILVA, T. C.; ALVES, R. R. $\mathrm{N}$. The current status of ethnobiological research in Latin America: gaps and perspectives. Journal of ethnobiology and ethnomedicine, v. 9, p. 1-9, jan. 2013.

AYRES, J. M.; FONSECA, G. A. B.; RYLANDS, A. B.; QUEIROZ, H. L.; PINTO, L. P.; MASTERSON, D.; CAVALCANTI, R. B. Os Corredores Ecológicos das Florestas Tropicais do Brasil. Belém: Sociedade Civil Mamirauá, 2005.

BAILEY, R. G. Ecoregions: the Ecosystem Geography of the Oceans and Continents. 2a. ed. New York: Springer, 2014.

BENSUSAN, N. Conservação da Biodiversidade em áreas protegidas. Rio de Janeiro: Editora FGV, 2006.

BERNARD, E.; BARBOSA, L.; CARVALHO, R. Participatory GIS in a sustainable use reserve in Brazilian Amazonia: Implications for management and conservation. Applied Geography, v. 31, n. 2, p. 564-572, abr. 2011.

BOURGOIN, J. Sharpening the understanding of socio-ecological landscapes in participatory land-use planning. A case study in Lao PDR. Applied Geography, v. 34, p. 99-110, 2012.

BRASIL. Decreto $\mathbf{n}^{0}$ 23.793, de 23 de janeiro de 1934. Aprova o código florestal que com este baixa.Rio de Janeiro:Ministério da Agricultura, , 1934. Disponível em: <http://www.planalto.gov.br/ccivil_03/decreto/1930-1949/D23793impressao.htm>. Acesso em: 21 jan. 2015

Lei $\mathbf{n}^{0} 9.985$, de 18 de julho de 2000. Regulamenta o art. 225, § 10, incisos I, II, III e VII da Constituição Federal, institui o Sistema Nacional de Unidades de Conservação da Natureza e dá outras providências.BrasíliaMinistério do Meio Ambiente, , 2000. Disponível em: <http://www.planalto.gov.br/ccivil_03/leis/19985.htm>. Acesso em: 21 jan. 2015

Decreto $\mathrm{n}^{0}$ 5.758, de 13 de abril de 2006. Institui o Plano Estratégico Nacional de Áreas Protegidas - PNAP, seus princípios, diretrizes, objetivos e estratégias, e dá outras providências.Brasília, 2006. Disponível em: <http://www.planalto.gov.br/ccivil_03/_Ato20042006/2006/Decreto/D5758.htm>. Acesso em: 21 jan. 2015

BUIJS, A. E.; ARTS, B. J. M.; ELANDS, B. H. M.; LENGKEEK, J. Beyond environmental frames: The social representation and cultural resonance of nature in conflicts over a Dutch woodland. Geoforum, v. 42, n. 3, p. 329-341, jun. 2011. 
CHIMÈRE-DIAW, M. Escalas nas teorias da conservação: um outro conflito de civilizações? In: DIEGUES, A. C. S. (Ed.). . A ecologia política das grandes ONGs transnacionais conservacionistas. São Paulo: NUPAUB-USP, 2008. p. 105-124.

CROSBY, A. W. Imperialismo Ecológico: a expansão biológica da Europa 900 - 1900. Traducao José Augusto Ribeiro e Carlos Afonso Malferrari. São Paulo: Companhia de Bolso, 2011.

DAVENPORT, L.; RAO, M. The history of protection: paradoxes of the past and challenges for the future. In: TERBORGH, J.; SCHAIK, C. VAN; DAVENPORT, L.; RAO, M. (Eds.). . Making parks work: strategies for preserving tropical nature. Washington: Island Press, 2002. p. 66-101.

DIAMOND, J. M. The island dilemma: lessons of modern biogeographic studies for the design of natural reserves. Biological conservation, n. 7, p. 129-146, 1975.

DIEGUES, A. C. S. O mito moderno da natureza intocada. 6 ed. ampl ed. São Paulo: Hucitec: Nupaub-USP/CEC, 2008.

DUDLEY, N. Guidelines for applying protected area management categories. Gland, Switzerland: IUCN, 2008.

EKEN, G. et al. Key Biodiversity Areas as Site Conservation Targets. BioScience, v. 54, n. 12, p. 1110, 2004.

FORRESTER, J.; COOK, B.; BRECKEN, L.; CINDERBY, S.; DONALDSON, A. Combining participatory mapping with Q-methodology to map stakeholder perceptions of complex environmental problems. Applied Geography, v. 56, p. 199-208, 2015.

GALLINI, S. El ambiente entre representación y ecología. Varia Historia, v. 33, p. 76-104, 2005.

GOMEZ-POMPA, A.; KAUS, A. Taming the wilderness myth. BioScience, v. 42, n. 4, p. 271-279, 1992.

GUERRA, A. T.; COELHO, M. C. N. Unidades de Conservação: abordagens e características geográficas. Rio de Janeiro: Bertrand Brasil, 2009.

JORGE-PÁDUA, M. T. O Sistema Nacional de Unidades de Conservação. In: MEDEIROS, R.; ARAÚJO, F. F. S. (Eds.). . Dez anos do Sistema Nacional de Unidades de Conservação da Natureza: lições do passado, realizações presentes e perspectivas para o futuro. Brasília: MMA, 2011. p. 21-36.

LANGHAMMER, P. F.; BAKARR, M. I.; BENNUN, L. A.; BROOKS, T. M.; CLAY, R. P.; DARWALL, W.; SILVA, N. DE; EDGAR, G. J.; EKEN, G.; FISHPOOL, L. D. C.; FOSTER, M. N. Identification and Gap Analysis of Key Biodiversity Areas. Gland/Suiça: IUCN, 2007.

LIMA, R. P. N.; LUNA, T. I.; QUINHÕES, T. A. T.; OLIVEIRA, V. P. DE; CADEMARTORI, E. G.; FIGUEIREDO, R. P. DE; PEREIRA, J. L.; FONSECA, D. S.; IVO, J. B. DE O.; PAIVA, A. S. DE. Contribuições do projeto Corredores Ecológicos ao Sistema Nacional de Unidades de Conservação. In: LIMA, R. P. N.; LUNA, T. I.; QUINHÕES, T. A. T.; OLIVEIRA, V. P. DE; CADEMARTORI, E. G.; FIGUEIREDO, R. P. DE; PEREIRA, J. L.; FONSECA, D. S.; IVO, J. B. DE O.; PAIVA, A. S. DE (Eds. .) . Dez anos do Sistema Nacional de Unidades de Conservação da Natureza: lições do passado, realizações presentes e perspectivas para o futuro. Brasília: MMA, 2011. p. 115-129.

MARGULES, C. R.; PRESSEY, R. L. Systematic conservation planning. Nature, v. 405, n. 6783, p. 243-253, 11 maio 2000.

MEDEIROS, R. Evolução das tipologias e categorias de áreas protegidas no Brasil. Ambiente \& Sociedade, v. 9, n. 1, p. 41-64, 2006.

MEDEIROS, R.; GARAY, I. Singularidades do Sistema de Áreas Protegidas para a Conservação e Uso da Biodiversidade Brasileira. In: BECKER, B. K.; GARAY, I. (Eds.). . Dimensões Humanas da Biodiversidade: O Desafio de Novas Relações Sociedade-Natureza. [s.1.] Vozes, 2006. p. 159-184.

MEDEIROS, R.; YOUNG, C. E. F. Contribuição das Unidades de Conservação Brasileiras para a economia nacional. Brasília: [s.n.].

MORSELlO, C. Áreas protegidas públicas e privadas: seleção e manejo. 2a. ed. São Paulo: Annablume, FAPESP, 2006.

MYERS, N. Biodiversity Hotspots Revisited. BioScience, v. 53, n. 10, p. 916-917, 2003. 
MYERS, N.; MITTERMEIER, R. A.; MITTERMEIER, C. G.; FONSECA, G. A. B.; KENT, J. Biodiversity hotspots for conservation priorities. Nature, v. 403, n. February, p. 853-859, 2000.

OLSON, D. M. et al. Terrestrial Ecoregions of the World: A New Map of Life on Earth. BioScience, v. 51, n. 11, p. 933-938, 2001.

OLSON, D. M.; DINERSTEIN, E. The Global 200: a representation approach to conserving the Earth's most biologically valuable ecoregions. Conservation Biology, v. 12, n. 3, p. 502-515, 1998.

PÁDUA, J. A. Um sopro de destruição: pensamento político e crítica ambiental no Brasil escravista (1786 - 1888). 2a. ed. Rio de Janeiro: Jorge Zahar Ed., 2004.

POSSEY, D. A.; OVERAL, W. L. Ethnobiology: implications and aplications (D. A. POSSEY \& W. L. OVERAL, Eds.)Proceedings of the First International Congress of Ethnobiology. Belém, 1988. Anais...Belém: Museu Paraense Emílio Goeldi, 1990

PRESSEY, R. L. Ad hoc reservations: forward or backward steps in developing representative reserve systems? Conservation biology, v. 8, n. 3, p. 6620-668, 1994.

PRESSEY, R. L.; HUMPHRIES, C. J.; MARGULES, C. R.; VANE-WRIGHT, R. I.; WILLIAMS, P. H. Beyond opportunism: Key principles for systematic reserve selection. Trends in ecology \& evolution, v. 8, n. 4, p. 124-8, abr. 1993.

RAMBALDI, G.; MUCHEMI, J.; CRAWHALL, N.; MONACI, L. Through the Eyes of HunterGatherers: participatory 3D modelling among Ogiek indigenous peoples in Kenya. Information Development, v. 23, n. 2-3, p. 113-128, 1 maio 2007.

REIGOTA, M. Meio Ambiente e Representação Social. 8a. ed. São Paulo: Cortez, 2010.

RODRIGUES, A. S. L. et al.Global gap analysis: towards a representative network of protected areas. Washington: Conservation International, 2003. v. 5

RYLANDS, A. B.; BRANDON, K. Unidades de conservação brasileiras. Megadiversidade, v. 1, n. 1, p. 27-35, 2005.

SCHERL, L. M.; WILSON, A.; WILD, R.; BLOCKHUS, J.; FRANKS, P.; MCNEELY, J. A.; MCSHANE, T. O. As áreas protegidas podem contribuir para a redução da pobreza? Oportunidades e limitações. Gland/Suiça e Cambridge/Reino Unido: IUCN, 2006.

SIEBER, R. Public participation geographic information systems: A literature review and framework. Annals of the Association of Geographers, v. 96, n. January, p. 491-507, 2006.

SODHI, N. S.; LEE, T. M.; SEKERCIOGLU, C. H.; WEBB, E. L.; PRAWIRADILAGA, D. M.; LOHMAN, D. J.; PIERCE, N. F.; DIESMOS, A. C.; RAO, M.; EHRLICH, P. R. Local people value environmental services provided by forested parks. Biodiversity Conservation, v. 19, n. 4, p. 11751188, 17 nov. 2010.

SULlivAN, A. L.; SHAFFER, M. L. Biogeography of the Megv. Science, v. 189, n. 4196, p. 13-17, 1975.

TERBORGH, J.; SCHAIK, C. VON. Why the World Needs Parks. In: TERBORGH, J.; SCHAIK, C. VAN (Eds.). . Making Parks Work: Strategies for Preserving Tropical Nature. Washington: Island Press, 2002. p. 21-39.

WEINER, D.; HARRIS, T. M.; CRAIG, W. J. Community Participation and Geographical Information Systems. London: Taylor and Francis, 2002. 